THE ASTROPHYSICAL JOURNAL, 542:785-788, 2000 October 20

\title{
A NATURAL FORMALISM FOR MICROLENSING
}

\author{
ANDREW GOULD \\ Department of Astronomy, Ohio State University, 140 West 18th Avenue, Columbus, OH 43210; gould@astronomy.ohio-state.edu \\ Received 2000 January 24 ; accepted 2000 June 1
}

\begin{abstract}
If the standard microlensing geometry is inverted so that the Einstein ring is projected onto the observer plane rather than the source plane, then the relations between the observables $\left(\theta_{\mathrm{E}}, \tilde{r}_{\mathrm{E}}\right)$ and the underlying physical quantities $\left(M, \pi_{\mathrm{rel}}\right)$ become immediately obvious. Here $\theta_{\mathrm{E}}$ and $\tilde{r}_{\mathrm{E}}$ are the angular and projected Einstein radii, $M$ is the mass of the lens, and $\pi_{\text {rel }}$ is the lens-source relative parallax. I recast the basic formalism of microlensing in light of this more natural geometry and in terms of observables. I then find that the relations between observable and physical quantities assume an exceptionally simple form. In an appendix, I propose a set of notational conventions.
\end{abstract}

Subject headings: astrometry — gravitational lensing

\section{INTRODUCTION}

The geometry of point-lens microlensing (Einstein 1936; Refsdal 1964; Paczyński 1986) is so simple that students can derive all the basic results in a few hours. Nonetheless, this geometry has never been boiled down to its essence: the relationship between the underlying physical quantities and the observables. In particular, the "Einstein ring radius" $r_{\mathrm{E}}$, a central concept in the usual formulation, is not directly observable and has not been observationally determined for even one of the $\sim 500$ microlensing events observed to date. There appear to be three reasons that the natural geometric formulation has not been developed. First, the standard geometry is already so trivial that further simplification has not seemed worthwhile. Second, the theory of microlensing was already quite developed before it was realized what the observables were, and until very recently the prospects were poor for measuring these observables except in a handful of events. Third, the original impulse to microlensing searches was to probe the dark matter. This focused attention on the optical depth (a statistical statement about the ensemble of events) and secondarily on the Einstein timescale $t_{\mathrm{E}}$, which, of the three observables, is the one that has the most convoluted relation to the underlying physical parameters.

However, with the prospect of astrometric microlensing it is now possible that a second observable, the angular Einstein radius $\theta_{\mathrm{E}}$, will be routinely measured (Boden, Shao, \& Van Buren 1998; Paczyński 1998). Moreover, if these astrometric measurements are carried out by the Space Interferometry Mission (SIM) in solar orbit, then comparison of photometry from SIM and the ground will yield a third observable, the projected Einstein radius $\tilde{r}_{\mathrm{E}}$ (Refsdal 1966; Gould 1995; Gould \& Salim 1999). Hence, it is now appropriate to reformulate the microlensing problem in terms of these observables.

\section{GEOMETRY}

The upper panel of Figure 1 shows the standard presentation of microlensing geometry (e.g., Fig. 3 from Gould 1996). The observer (O), lens (L) of mass $M$, and source (S) are aligned. The light is deflected by an angle $\alpha$ given by the Einstein (1936) formula

$$
\alpha=\frac{4 G M}{r_{\mathrm{E}} c^{2}}
$$

where $r_{\mathrm{E}}$ is the Einstein radius. It arrives at the observer displaced by an angle $\theta_{\mathrm{E}}$ from the true position of the source. In this case, the source is therefore imaged into a ring. The size of this ring projected onto the source plane is $\hat{r}_{\mathrm{E}}$. More generally, the alignment will not be perfect, and the axial symmetry will be broken. Hence, there will be two images rather than a ring. However, even in this more general case the Einstein ring provides a natural scale to the problem.

The lower panel of Figure 1 basically inverts the geometry of the upper panel and thereby focuses attention on the observer rather than the source. This seems like a trivial change, but it has two advantages. First, the quantities shown at the right, $\theta_{\mathrm{E}}$ and $\tilde{r}_{\mathrm{E}}$, are the observables. To date, $\theta_{\mathrm{E}}$ has been measured for only eight events (Alcock et al. 1997, 2000; Albrow et al. 1999, 2000a, 2000b; Afonso et al. 2000), all by using the source as an "angular ruler" (Gould 1994a; Nemiroff \& Wickramasinghe 1994; Witt \& Mao 1994). Similarly, $\tilde{r}_{\mathrm{E}}$ has been determined for only about a half-dozen events (Alcock et al. 1995; Bennett et al. 1997; Mao 1999). For all of these, $\tilde{r}_{\mathrm{E}}$ was found by measuring the deviation of the light curve induced by the Earth's motion (Gould 1992). The amplitude of this deviation is proportional to $\pi_{\mathrm{E}} \equiv \mathrm{AU} / \tilde{r}_{\mathrm{E}}$. The measurements of both $\theta_{\mathrm{E}}$ and $\tilde{r}_{\mathrm{E}}$ have required special conditions (a caustic crossing for $\theta_{\mathrm{E}}$ and an event lasting a large fraction of a year for $\tilde{r}_{\mathrm{E}}$ ), which is why so few of these "observables" have actually been observed. However, as mentioned above, both $\theta_{\mathrm{E}}$ and $\tilde{r}_{\mathrm{E}}$ could be measured routinely in the future.

The second reason for inverting the standard geometry is that doing so makes transparent the relation between the observables and the underlying physical variables: the product of $\theta_{\mathrm{E}}$ and $\tilde{r}_{\mathrm{E}}$ is essentially the Schwarzschild radius of the lens, and their ratio is essentially the lens-source relative parallax. Using the small-angle approximation, one sees immediately from the lower panel of Figure 1 that $\alpha / \tilde{r}_{\mathrm{E}}=\theta_{\mathrm{E}} / r_{\mathrm{E}}$, or

$$
\theta_{\mathrm{E}} \tilde{r}_{\mathrm{E}}=\alpha r_{\mathrm{E}}=\frac{4 G M}{c^{2}}
$$

Next, from the exterior-angle theorem,

$$
\theta_{\mathrm{E}}=\alpha-\psi=\frac{\tilde{r}_{\mathrm{E}}}{D_{l}}-\frac{\tilde{r}_{\mathrm{E}}}{D_{s}}=\frac{\tilde{r}_{\mathrm{E}}}{D_{\text {rel }}}
$$



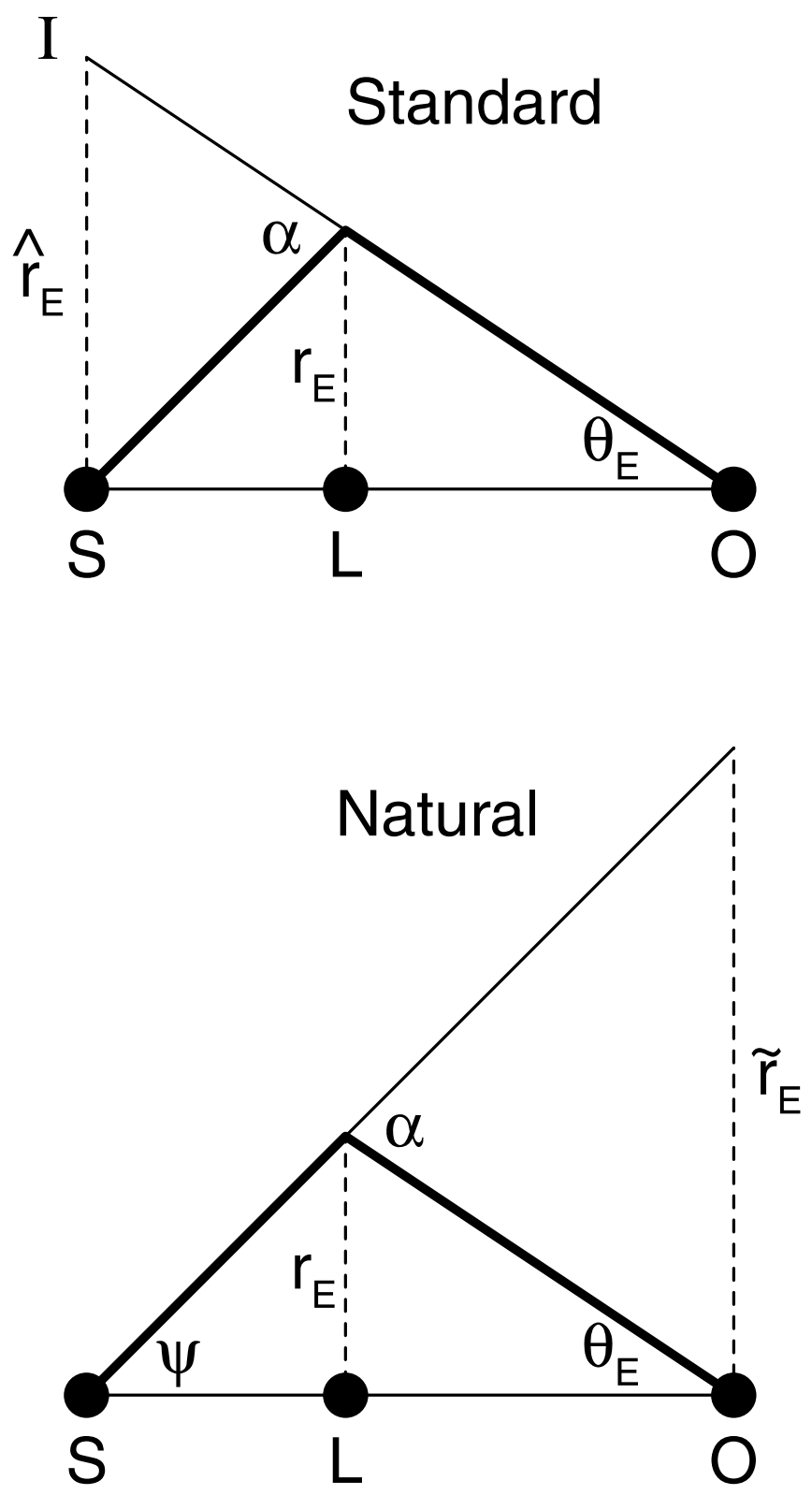

Fig. 1.-Above: Standard microlensing geometry. The bold curve shows the path of the light from the source $(\mathrm{S})$ to the observer $(\mathrm{O})$ being deflected by the lens (L) of mass $M$. The deflection angle is $\alpha=4 G M / r_{\mathrm{E}} c^{2}$, where $r_{\mathrm{E}}$ is the Einstein radius shown as a dashed line. The image (I) is displaced from the source by the angular Einstein radius $\theta_{\mathrm{E}}$, which, projected onto the source plane, corresponds to a physical distance $\hat{r}_{\mathrm{E}}$. Below: Natural microlensing geometry. Mostly the same as the upper panel, except that the Einstein radius is now projected onto the observer plane as $\tilde{r}_{\mathrm{E}}$ rather than onto the source plane as $\hat{r}_{\mathrm{E}}$. This minor difference allows one to see immediately the relations between the observables $\left(\theta_{\mathrm{E}}, \tilde{r}_{\mathrm{E}}\right)$ and the physical parameters $\left(M, \pi_{\text {rel }}\right)$. First, under the small-angle approximation, $\alpha / \tilde{r}_{\mathrm{E}}=\theta_{\mathrm{E}} / r_{\mathrm{E}}$, so $\tilde{r}_{\mathrm{E}} \theta_{\mathrm{E}}=\alpha r_{\mathrm{E}}=4 G M / c^{2}$. Second, by the exterior-angle theorem, $\theta_{\mathrm{E}}=\alpha-\psi=\tilde{r}_{\mathrm{E}} / D_{l}-\tilde{r}_{\mathrm{E}} / D_{s}$, where $D_{l}$ and $D_{s}$ are the distances to the lens and source. Hence, $\theta_{\mathrm{E}} / \tilde{r}_{\mathrm{E}}=\pi_{\mathrm{rel}} / \mathrm{AU}$, where $\pi_{\text {rel }}$ is the lens-source relative parallax.

where $D_{l}$ and $D_{s}$ are the distances to the lens and source, and $D_{\text {rel }}^{-1} \equiv D_{l}^{-1}-D_{s}^{-1}$. Note that equation (3) can be written more suggestively as

$$
\pi_{\mathrm{E}} \theta_{\mathrm{E}}=\pi_{\mathrm{rel}}, \quad \pi_{\mathrm{E}} \equiv \frac{\mathrm{AU}}{\tilde{r}_{\mathrm{E}}},
$$

where $\pi_{\text {rel }}=\mathrm{AU} / D_{\text {rel }}$ is the lens-source relative parallax.
Just as in astrometric parallax determinations, where $\pi$ is a more natural way to represent the measured quantity than its inverse (distance), so in microlensing "parallax" determinations $\pi_{\mathrm{E}}$ is more natural than its inverse $\left(\tilde{r}_{\mathrm{E}}\right)$. The reason is the same: the observable effect is inversely proportional to $\tilde{r}_{\mathrm{E}}$ but directly proportional to $\pi_{\mathrm{E}}$, so the measurement errors when expressed in terms of $\pi_{\mathrm{E}}$ exhibit more regular behavior. As in the case of astrometric parallax, this feature becomes especially important for measurements that are consistent with zero at the few- $\sigma$ level. Indeed, in contrast to astrometric parallaxes, microlensing parallaxes are inherently two-dimensional (Gould 1995). That is, one measures not only the amplitude of $\tilde{r}_{\mathrm{E}}\left(\right.$ or $\pi_{\mathrm{E}}$ ) but also the direction of lens-source relative motion. Hence one can generalize $\pi_{\mathrm{E}}$ to a two-dimensional vector $\pi_{\mathrm{E}}$ whose direction is that of the lens relative to the source. The measurement errors in $\pi_{\mathrm{E}}$ are then easily expressed as a covariance matrix. By contrast, there is no natural way to generalize $\tilde{r}_{\mathrm{E}}$ : it can be made into a vector with the same direction $\tilde{r}_{\mathrm{E}}$, but when $\pi_{\mathrm{E}}$ is consistent with zero, such a vector is very poorly behaved. Moreover, in some cases one component of $\pi_{\mathrm{E}}$ can be very well determined while the other is highly degenerate (Refsdal 1966; Gould 1994b, 1995), a situation that is easily represented using $\pi_{\mathrm{E}}$ but unwieldy using $\tilde{\boldsymbol{r}}_{\mathrm{E}}$. (Note that while no one has ever previously introduced the vector $\tilde{r}_{\mathrm{E}}$, I have often discussed the closely related projected velocity vector, $\tilde{v}=\tilde{\boldsymbol{r}}_{\mathrm{E}} / t_{\mathrm{E}}$.)

The Einstein crossing time $t_{\mathrm{E}}$ is the only observable that at present is routinely observed. While I find no fault with $t_{\mathrm{E}}$, considerations of symmetry with the substitution $\tilde{r}_{\mathrm{E}} \rightarrow \pi_{\mathrm{E}}$ lead me to substitute $t_{\mathrm{E}} \rightarrow \mu_{\mathrm{E}}$, where

$$
\mu_{\mathrm{E}} \equiv \frac{1}{t_{\mathrm{E}}},
$$

and where the direction of $\mu_{\mathrm{E}}$ is that of the lens motion relative to the source. With this definition, the relative lenssource proper motion is given by $\mu_{\mathrm{rel}}=\mu_{\mathrm{E}} \theta_{\mathrm{E}}$.

\section{RELATIONS BETWEEN OBSERVABLES AND PHYSICAL QUANTITIES}

From equations (2)-(4), one immediately derives

$$
\tilde{r}_{\mathrm{E}}=\sqrt{\frac{4 G M D_{\mathrm{rel}}}{c^{2}}}, \quad \pi_{\mathrm{E}}=\sqrt{\frac{\pi_{\mathrm{rel}}}{\kappa M}}
$$

and

$$
\theta_{\mathrm{E}}=\sqrt{\frac{4 G M}{D_{\text {rel }} c^{2}}}=\sqrt{\kappa M \pi_{\text {rel }}},
$$

where

$$
\kappa \equiv \frac{4 G}{c^{2} \mathrm{AU}}=\frac{4 v_{\oplus}^{2}}{M_{\odot} c^{2}} \simeq 8.144 \frac{\mathrm{mas}}{M_{\odot}},
$$

and $v_{\oplus} \sim 30 \mathrm{~km} \mathrm{~s}^{-1}$ is the speed of the Earth.

How well is the coefficient $(8.14 \ldots)$ in $\kappa$ known? It suffers from two sources of uncertainty. First, the factor 4 in equations (8) and (1) is a prediction of general relativity (GR). Its accuracy (often parameterized by $\gamma$ ) has been verified experimentally by Hipparcos, but only to $0.3 \%$ (Froeschle, Mignard, \& Arenou 1997). However, if GR is assumed to be exact, then this coefficient can be determined as accurately as $\left(v_{\oplus} / c\right)^{2}$, which should be known from pulsar 
timing and solar system radar ranging to at least nine significant digits.

In astrometric microlensing measurements, one automatically recovers the parallax and proper motion of the source, $\pi_{s}$ and $\boldsymbol{\mu}_{s}$ (Boden et al. 1998; Gould \& Salim 1999). Hence, the observables are $\mu_{\mathrm{E}}, \pi_{\mathrm{E}}, \theta_{\mathrm{E}}, \pi_{s}$ and $\mu_{s}$. When expressed in this natural form, they have a particularly simple relation to the physical properties of the lens:

$$
\begin{gathered}
M=\frac{\theta_{\mathrm{E}}}{\kappa \pi_{\mathrm{E}}}, \\
\pi_{l}=\pi_{\mathrm{E}} \theta_{\mathrm{E}}+\pi_{s},
\end{gathered}
$$

$$
\boldsymbol{\mu}_{l}=\boldsymbol{\mu}_{\mathrm{E}} \theta_{\mathrm{E}}+\boldsymbol{\mu}_{\mathrm{E}}
$$

and

$$
\boldsymbol{v}_{\perp, l}=\frac{\mu_{\mathrm{E}} \theta_{\mathrm{E}}+\boldsymbol{\mu}_{s}}{\pi_{\mathrm{E}} \theta_{\mathrm{E}}+\pi_{s}},
$$

where $\pi_{l}, \mu_{l}$, and $v_{\perp, l}$ are the parallax, proper motion, and transverse velocity of the lens.

I thank A. Babul, D. Bennett, N. Dalal, K. Griest, E. Kerins, and S. Rhie for useful comments and clarifications. This work was supported by grant AST 97-27520 from the NSF.

\section{APPENDIX}

\section{THE NEED FOR UNIFORM NOTATION}

Microlensing suffers from a plethora of mutually inconsistent notational conventions. While this poses no real problem for veterans, it presents significant obstacles to newcomers entering the field. I take the opportunity of this paper (which, more than most, concerns itself with notational issues) to try to forge a consensus. In formulating my proposed conventions, I am influenced primarily by prevalence of current usage and secondarily by the need for internal consistency.

I am abandoning some of my own prized notations, and I hope others are willing to do the same in the interest of achieving a uniform system. A number of comments I received after a preprint of this paper appeared give grounds for cautious optimism.

First, all quantities associated with the size of the Einstein ring (in units of length, angle, time, etc.) should be subscripted with an uppercase roman " $\mathrm{E}$ " in conformity with ApJ conventions. All physical Einstein radii should be denoted $r$. Hence, $\tilde{r}_{\mathrm{E}}$, $r_{\mathrm{E}}$, and $\hat{r}_{\mathrm{E}}$ for the Einstein rings in the planes of the observer, lens, and source. The other quantities are $\theta_{\mathrm{E}}$ for the angular Einstein radius, $t_{\mathrm{E}}$ for the Einstein radius crossing time, $\mu_{\mathrm{E}} \equiv t_{\mathrm{E}}^{-1}, \pi_{\mathrm{E}} \equiv \mathrm{AU} / \tilde{r}_{\mathrm{E}}$, and the direction of $\pi_{\mathrm{E}}$ and $\mu_{\mathrm{E}}$ defined by the direction of the proper motion of the lens relative to the source. Note that the Einstein diameter crossing time is by common convention denoted $\hat{t}$.

Second, all quantities associated with position in the Einstein ring should be denoted by $u$, or possibly by $\boldsymbol{u}$ if a vector position is indicated. When $\boldsymbol{u}$ is a vector, it must be specified whether it is the source position relative to the lens or vice versa. Common usage seems to conform to the former, and hence I adopt that. However, keep in mind that this means that $d u / d t=-\boldsymbol{\mu}_{\mathrm{rel}} / \theta_{\mathrm{E}}$.

Third, all quantities associated with the time of closest approach to the center of the Einstein ring should be denoted by a subscript zero: thus, $t_{0}$ for the time of closest approach and $u_{0}$ for the projected separation of the lens and source in units of $\theta_{\mathrm{E}}$ at time $t_{0}$.

Fourth, all quantities associated with the source should be denoted by a subscript asterisk: thus, $\theta_{*}$ for the angular radius of the source and $r_{*}$ for its physical radius.

Fifth, time normalized to the Einstein crossing time should be denoted $\tau_{\mathrm{E}}=\left(t-t_{0}\right) / t_{\mathrm{E}}$. Hence the vector position in the Einstein ring is $\boldsymbol{u}=\left(\tau_{\mathrm{E}}, u_{0}\right)$.

Sixth, the distances to the lens and source should be denoted $D_{l}$ and $D_{s}$.

Seventh, event parameters as measured from locations other than the Earth should be subscripted, e.g." " $t_{0, s}$ " for the time of closest approach as seen from a satellite. The subscript Sun symbol $(\odot)$ should be reserved for event parameters as seen from the Sun (not in the Sun frame but from another location). The subscript Earth symbol $(\oplus)$ should be used only when needed to avoid confusion.

Finally, the reader will note that I have described different parameters that contain the same information, e.g., $\left(\tilde{r}_{\mathrm{E}}, \pi_{\mathrm{E}}\right)$ and $\left(t_{\mathrm{E}}, \mu_{\mathrm{E}}\right)$. I expect that $\pi_{\mathrm{E}}$ and $\mu_{\mathrm{E}}$ will come into use mainly in technical applications, and that the general reader of microlensing articles will continue to find $\tilde{r}_{\mathrm{E}}$ and $t_{\mathrm{E}}$ to be more intuitive. In particular, in cases where there is only a microlensing parallax measurement, the projected velocity $\tilde{\boldsymbol{v}}=\mu_{\mathrm{E}} / \pi_{\mathrm{E}}$ is often a substantially more useful representation of the measurement than $\mu_{\mathrm{E}}$ and $\pi_{\mathrm{E}}$ reported separately. Note that in contrast to $v_{l, \perp}=\mu_{l} / \pi_{l}$, which represents two components of an intrinsically three-dimensional vector, $\tilde{v}$ is intrinsically two-dimensional and so should not be subscripted with a "perpendicular" symbol $(\perp)$.

Afonso, C., et al. 2000, ApJ, 532, 340

Albrow, M., et al. 1999, ApJ, 522, 1011 2000a, ApJ, 534, 894

2000b, ApJ, submitted (astro-ph/0004243)

Alcock, C., et al. 1995, ApJ, 454, L125

. 1997, ApJ, 491, 436

\section{REFERENCES}

Alcock, C., et al. 2000, ApJ, 541, 270

Bennett, D. B., et al. 1997, BAAS, 191.8303

Boden, A. F., Shao, M., \& Van Buren, D. 1998, ApJ, 502, 538

Einstein, A. 1936, Science, 84, 506

Froeschle, M., Mignard, F., \& Arenou, F. 1997, Proc. ESA Symp., Hipparcos—Venice '97 (ESA SP-402; Paris: ESA), 49 


\section{GOULD}

Gould, A. 1992, ApJ, 392, 442

1994a, ApJ, 421, L71

1994b, ApJ, 421, L75

1995, ApJ, 441, L21

1996, PASP, 108, 465

Gould, A., \& Salim, S. 1999, ApJ, 524, 794

Mao, S. 1999, A\&A, 350, L19
Nemiroff, R. J., \& Wickramasinghe, W. A. D. T. 1994, ApJ, 424, L21

Paczyński, B. 1986, ApJ, 304, 1

1998, ApJ, 494, L23

Refsdal, S. 1964, MNRAS, 128, 295

1966, MNRAS, 134, 315

Witt, H. J., \& Mao, S. 1994, ApJ, 429, 66 